HORMONE-RECEPTOR INTERACTIONS: A STUDY OF THE BINDING OF HORMONE-SUBSTITUTED TOBACCO MOSAIC VIRUS TO MEMBRANE VESICLES BY DYNAMIC LIGHT SCATTERING AND BY TRANSIENT ELECTRIC BIREFRINGENCE

Robert Schwyzer, V. Marly Kriwaczek

Institut für Molekularbiologie und Biophysik

Kurt Baumann, Hans-Ruedi Haller, Gerhard Wider, and Pierre Wiltzius

Laboratorium für Festkörperphysik, Eidgenössische Technische Hochschule, CH-8093 zürich, Switzerland

\begin{abstract}
Tobacco mosaic virus has been covalently conjugated with 500 600 molecules of different hormones. The particles react with antisera against the hormones and with hormone receptors in target organs, on target cells, and on membrane vesicles. The compounds were used for cooperative affinity labelling and for the isolation of receptor-bearing vesicles. The binding of a tobacco mosaic virus/angiotensin II conjugate to adrenal cortex membrane vesicles was studied by dynamic light scattering and transient electric birefringence. Despite the complexity of the polydisperse systems and the fact that the membrane vesicles exhibit a Kerr effect and depolarize the light, methods were developed that allowed the observation and theoretical treatment of the effects due to specific binding.
\end{abstract}

\title{
INTRODUCTION
}

Tobacco mosaic virus (TMV) is an easily accessible, crystalline nucleoprotein with a molecular weight of about 40,000,000 Daltons. The virus particle is a hollow cylinder (diameter $180 \AA$, length $3,000 \AA$ ) consisting of a ribonucleic acid genome and a capsid of 2,130 identical polypeptide capsomers. The $\varepsilon$-amino groups of the lysine residues No. 68 of each capsomer are the only amino groups available for substitution in the intact virus (for a recent review of TMV structure and chemistry, see Ref. 1).

In order to study hormone-receptor interactions, to localize hormone receptors, and to facilitate receptor isolation, we have prepared covalent conjugates between TMV and hormones. About 500 - 600 amino groups per virus particle were substituted with mercaptopropionic acid and the introduced mercapto groups reacted with biologically active derivatives of bromoacetyl $\alpha$-melanotropin and $\varepsilon$-maleiimidohexanoyl angiotensin II to produce 'TMV-S-hormone' (Ref. 2 \& 3).

The compounds were biologically active, were aggregated by hormone antisera, and strongly bound by receptor-bearing membrane vesicles from target organs. The latter reaction allowed the separation of TMV-S-hormone/vesicle aggregates from unreacted virus particles and vesicles by density gradient centrifugation (Ref. 3). All of the observations suggested an especially strong, specific association between TMV-S-hormone and receptors on the plasma membrane.

We have interpreted this phenomenon as possibly arising from cooperative affinity interactions (Ref. 2 \& 3). A rough calculation shows that the TMV-hormone particle is large

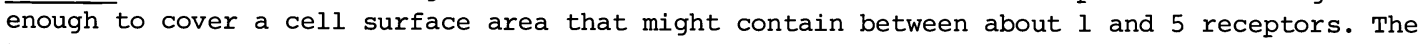
hormone molecules attached to a single virus would then be able to react simultaneously with more than one receptor, resulting in a potentiation of the over-all association constant. (The situation is that of a polyvalent ligand reacting with a polyvalent acceptor.)

The physical studies reported here in a preliminary manner support this view. The binding of a (3-mercapto-1-succinyl)-TMV/ $\varepsilon$-maleimidohexanoyl-[1-asparagine,5-valine] angiotensin II conjugate (TMV-S-ANG') to bovine adrenal cortex cell membrane vesicles containing angiotensin 
binding sites (VESICLES) (Ref. 3) was studied by dynamic light scattering (Ref. 4) and signal-averaging transient electric birefringence (Ref. 5; for more recent literature, see Ref.6). The results shall be published in detail elsewhere.

\section{DYNAMIC LIGHT SCATTERING}

Our original expectations of the system TMV-S-ANG'/VESICLES were: (i) Vesicles as roughly spherical particles will not depolarize the incident light. (ii) The rotational diffusion constants of 'free' and 'bound' virus particles should be markedly different $\left(D_{R}^{F}>D_{R}^{B}\right)$. Hence, the titration of TMV-S-ANG' with VESICLES should cause the appearance of ${ }^{R}$ ' 'slow' component in the intensity autocorrelation function. However, it was found that vesicles do depolarize light and that our TMV, especially TMV-S-ANG' preparations, already contain about 25 of a 'slow' component (Fig. 1).

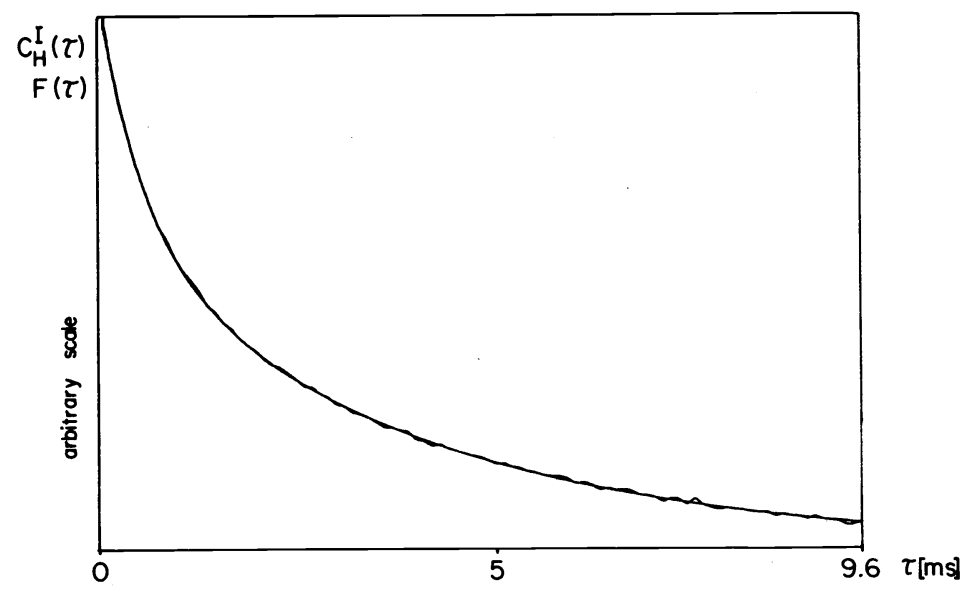

Fig. 1. Depolarized intensity autocorrelation function, $\mathrm{C}_{\mathrm{H}}^{\mathrm{I}}(\tau)$, obtained with the heterodyne method for a TMV preparation containing $0.5 \mathrm{~g} / 1$ of TMV in $1 \mathrm{mM}$ HEPES buffer ('noisy' curve). Smooth curve, $F(\tau)$, fitted to $C_{H}^{I}(\tau)$ with 2 exponentials, equation ( 1 ).

$$
\begin{aligned}
& F(\tau)=B_{1}+B_{2}\left[B_{3} \exp \left(-\tau / B_{4}\right)+\left(1-B_{3}\right) \exp \left(-\tau / B_{5}\right)\right] \\
& D_{R}^{1}=1 / 6 B_{4} \\
& D_{R}^{2}=1 / 6 B_{5}
\end{aligned}
$$

From the fitted curve parameters of (1), the rotatory diffusion constants were calculated according to equations (2) and (3). They correspond to unaggregated $T M V\left(D_{\bar{R}}^{7}=287 \mathbf{s}^{-1}\right.$, $1 \simeq 3000 \AA)$ and an end-to-end aggregated species $\left(D_{R}^{2}=43.4 \mathrm{~s}^{-1}, 1 \simeq 6000 \AA\right)$ that can also be seen by electron microscopy. The results agree roughly with those reported in the literature (e. $\underline{\text { g. }}$. Ref. 7 \& 8 ).

Similar measurements with VESICLES gave $D_{R}=20.4 \mathrm{~s}^{-1}$, which would correspond ideally to an assembly of spheres with diameters of about $450 \mathrm{~nm}$.

Because VESICLES also depolarize the incident light and because their rotational diffusion constants are so close to those of the slow components of TMV and TMV-S-ANG', it was 
impossible to devise an experiment that would 'see' only the virus particles. It was therefore decided to use the intensity decrease of the 'fast' virus component as a measure for binding. In the actual experiments, increasing amounts of VESICLES were admixed to TMV and TMV-S-ANG', and $\mathrm{B}_{3} / \mathrm{B}_{3}^{0}$ (ratio of the relative amplitude of the 'fast' component with VESICLES to the relative amplitude of the 'fast' component without VESICLES) plotted against the ratios of VESICLE/TMV or VESICLE/TMV-S-ANG', as in Fig. 2.

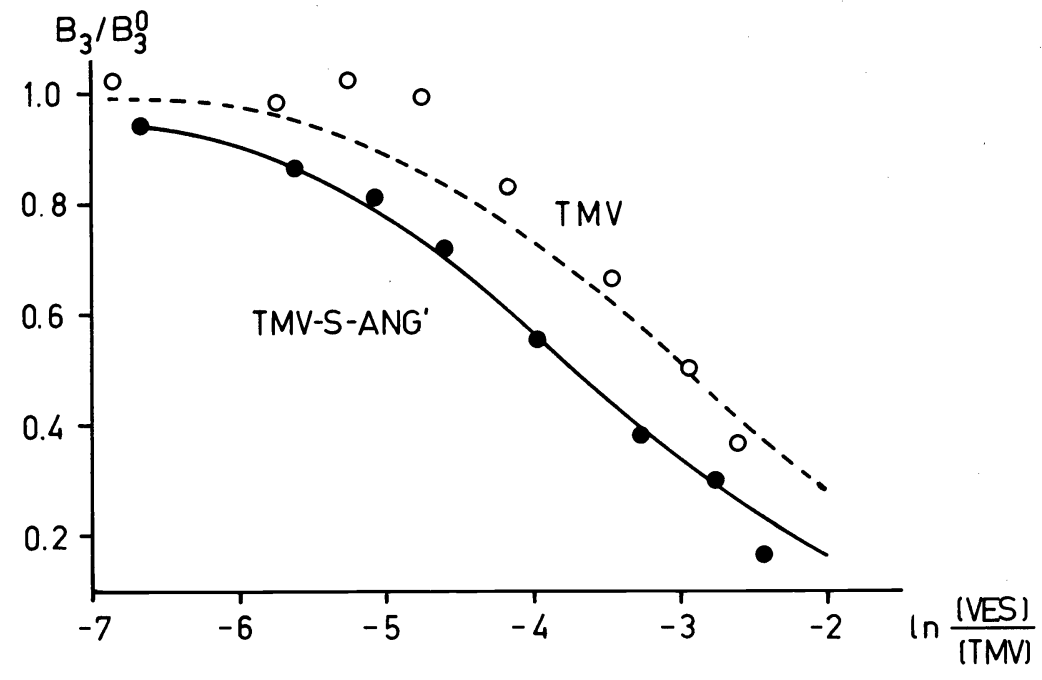

Fig. 2. Titration of TMV (o) and TMV-S-ANG' (•) with VESICLES. Calculated curves for no binding (----) and for the binding $(\longrightarrow$ of about 30 TMV-S-ANG' particles per VESICLE (see text).

The model that was developed to interpret these findings had to take into account that the intensity of the light scattered by a particle is proportional to the square of the particle volume. Therefore the intensity of a system consisting of one vesicle and several virus particles increases as soon as the virus particles become bound to the vesicle. Equations (4) and (5) were assumed to describe adequately the situations of 'no binding' and 'binding', respectively:

$$
\begin{aligned}
& \frac{B_{3}}{B_{3}^{0}}=\frac{1}{1+\varepsilon \frac{\left.<N_{v}\right\rangle}{\left\langle N_{t}\right\rangle}} \\
& \frac{B_{3}}{B_{3}^{0}}=\frac{1}{1+(\varepsilon+\gamma r) \frac{\left\langle N_{v}\right\rangle}{\left.\left\langle N_{t}\right\rangle-r<N_{v}\right\rangle}}
\end{aligned}
$$

Therein $\varepsilon$ is the ratio of the intensity produced by one 'free' VESICLE to that of one 'free' TMV or TMV-S-ANG' particle, $\gamma$ is the ratio of intensities of bound to free virus particles, $r$ the number of virus particles bound per vesicle, and $\left\langle\mathrm{N}_{\mathrm{v}}\right\rangle$ and $\left\langle\mathrm{N}_{\mathrm{t}}\right\rangle$ the number of vesicles and viruses, respectively, within the scattering volume.

With the help of the ratio $\left\langle\mathrm{N}_{\mathrm{v}}\right\rangle /\left\langle\mathrm{N}_{t}\right\rangle$ at the point $\mathrm{B}_{3} / \mathrm{B}_{3}=0.5$ (determined from the concentrations at that point of the TMV titration curve of Fig. 2), $\varepsilon$ was approximated (4180 in this, and 6640 in another example). Insertion of this value into (5) and similar treatment yielded an approximation for $r$. An estimate of $\gamma$ was obtained from $\varepsilon$ and $r$. The average number of TMV-S-ANG' bound per VESICLE, $r$, was found to be in the order of 30 (31 and 28 for the two experiments, respectively). 
Pulses of $48 \mathrm{~V}, 1 \mathrm{~ms}$, were used to produce a transient Kerr effect. Studies with our TMV preparations gave $\mathrm{D}_{\mathrm{R}}^{1}=(336 \pm 6.6) \mathrm{s}^{-1}$ and $\mathrm{D}_{\mathrm{R}}^{2}=(52.8 \pm 3.0) \mathrm{s}^{-1}$ corresponding to calculated cylinder lengths of about 300 and $600 \mathrm{~nm}$ (Ref. 9). The values for TMV-S-ANG' varied with the age of the sample. Just after preparation, they were very similar to those for TMV: $D_{R}^{l}=$

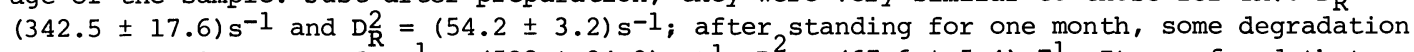
had apparently occurred: $D_{R}^{1}=(599 \pm 24.0) \mathrm{s}^{-1}, \mathrm{D}_{\mathrm{R}}^{2}=(67.6 \pm 5.4) \mathrm{s}^{-1}$. It was found that vesicles also exhibited electric birefringence. The observed decay curves were characteristic of a polydisperse system in agreement with the electron-microscopic findings (Ref. 10). They were approximated with two exponentials. The apparent rotational diffusion constants, " $D_{R} "$ (that are not necessarily caused by Brownian rotation alone) were: " $\mathrm{D}_{\mathrm{R}}^{1}=(474.0 \pm 28.5)$, $(543.5 \pm 27.0) \mathrm{s}^{-1}$ and $" \mathrm{D}_{\mathrm{R}}^{2} "=(26.8 \pm 3.2),(30.1 \pm 2.4) \mathrm{s}^{-1}$ for two different preparations. Measurement of the rotational diffusion constants of various mixtures of TMV or TMV-S-ANG' with VESICLES gave the results exemplified by the experiment of Fig. 3 .

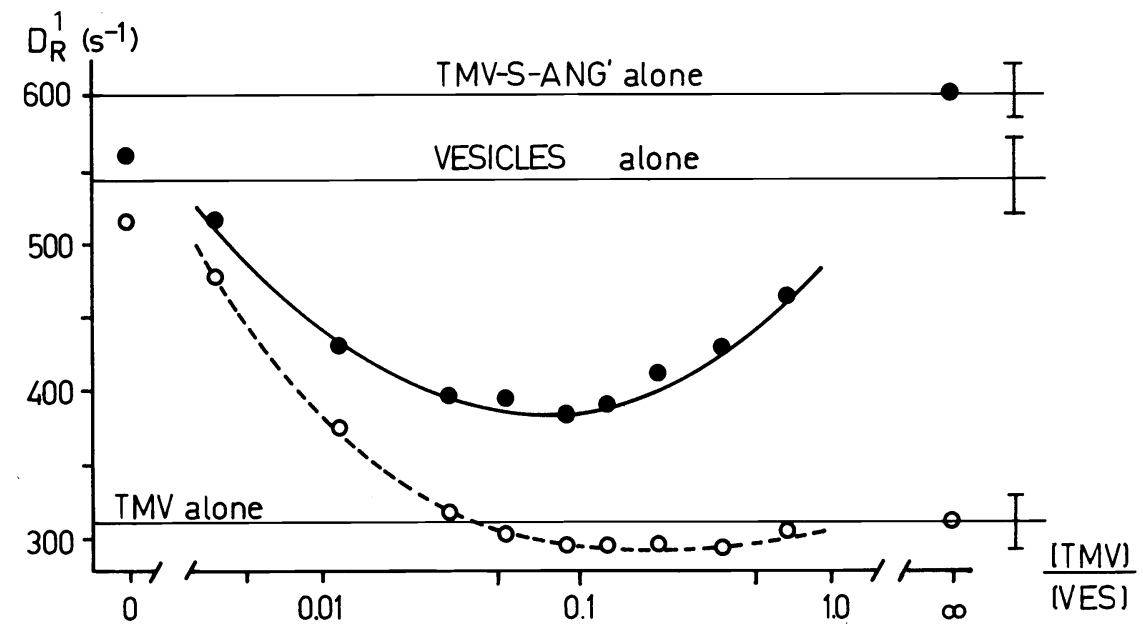

Fig. 3. Rotational diffusion constants of various mixtures of TMV (o) or TMV-S-ANG' ( $\bullet$ ) with VESICLES measured by signal-averaging transient electric birefringence. [TMV]/[VES] indicates the mixing ratio of solutions containing $0.12 \mathrm{~g} / 1$ of TMV or TMV-S-ANG' and $0.1 \mathrm{~g} / 1$ VESICLES, respectively, in $1 \mathrm{mM}$ HEPES buffer, pH 6.8. This corresponds to roughly $1.5 \cdot 10^{12}$ virus particles and $2 \cdot 10^{10}$ vesicles per $\mathrm{ml}$

The results were explained in the following manner: The values for " $D_{R}^{l}$ " (VESICLES) are changed to those for $D_{R}^{1}(T M V)$ or $D_{R}^{1}(T M V-S-A N G ')$ when these components are in excess. With native TMV the values decline rather rapidly because the signal produced by the virus is much more intense than that of the vesicle. The curves were not changed, if aged VESICLES were used that had lost most of their binding capacity (Ref. 10). This indicates that TMV is not bound appreciably.

The fact that aged TMV-S-ANG' has a somewhat larger $D_{R}^{1}$ than VESICLES proved to be very

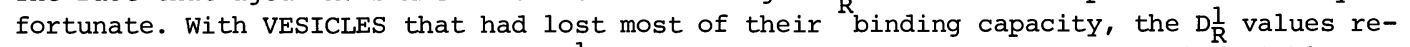
mained always between 550 and $600 \mathrm{~s}^{-1}$ if TMV-S-ANG' was admixed. However, with viable, angiotensin II-binding VESICLES the results shown in Fig. 3 were obtained. The distinct minimum indicates the appearance of more slowly relaxing particles, such as a VESICLE/TMV-S-ANG' complex. After saturation of the binding sites, $D_{R}^{1}$ begins to rise again as a result of the excess TMV-S-ANG'. The ratio of particles at the minimum gives an ideia of the mean number, $r$, of TMV-S-ANG' bound per VESICLE if it is assumed that binding is saturable and virtually irreversible. In this case, $r=8$. 


\section{DISCUSSION}

The strong, selective binding of a TMV/angiotensin II conjugate to bovine adrenal cortex membrane vesicles containing angiotensin II binding sites that had been observed earlier by density gradient centrifugation and electron microscopy (Ref. 3) has been confirmed by dynamic light scattering and signal-averaging transient electric birefringence. The polydispersity of the systems and the fact that vesicles depolarize the light and actually show a Kerr effect caused difficulties. These were overcome by developing a model for depolarized light scattering that takes into account the intensity increase caused by the binding of a virus particle to a vesicle. With transient electric birefringence, the appearance of components with longer relaxation times were observed directly. The results indicate that on the average $10-30 \mathrm{TMV} /$ angiotensin II conjugate particles are strongly bound per vesicle; the binding is much less with unsubstituted TMV or with aged vesicles that have lost their binding capacity for angiotensin II. These results suggest that the binding is caused by membrane components that are specific for angiotensin (receptors? other binding sites?) and do not contradict the idea of the interaction being due to cooperative affinity. The results are still semiquantitative and could be made more accurate by decreasing the polydispersity of the systems.

\footnotetext{
Acknowledgement - This work was supported by grants from the Swiss National Science Foundation. It is part of the doctoral thesis of H.-R. H.
}

\section{REFERENCES}

1. A.C.H. Durham and P.J.G. Butler, Eur.J.Biochem. 53, 397-404 (1975).

2. V.M. Kriwaczek, A.N. Eberle, M. Müller, and R. Schwyzer, Helv.Chim.Acta 61, 1232-1240 (1978) .

3. V.M. Kriwaczek, J.C. Bonnafous, M. Müller, and R. Schwyzer, Helv.Chim.Acta 61, 12411245 (1978).

4. B. Berne and R. Pecora, Dynamic Light Scattering, Wiley, New York (1976).

5. C.T. O'Konski, in Encyclopedia of Polymer Science and Technology 9, 551-590, Interscience, New York (1968).

6. J. Newman and H.L. Swinney, Biopolymers 15, 301-315 (1976).

7. J.M. Schurr and K. Schmitz, Biopolymers $\frac{12}{12}, 1021-1045$ (1973).

8. T. King, A. Knox, and J.D.G. McAdam, Biopolymers 12, 1917-1926 (1973).

9. S. Broersma, J.Chem.Phys. 32, 1626-1631 (1960).

10. W. Schlegel and R. Schwyzer, Eur.J.Biochem. 72, 415-424 (1977). 